\title{
Acompanhamento Terapêutico: Concepções e Possibilidades em Serviços de Saúde Mental
}

\author{
Amanda Candeloro Cunha \\ Danielle Abdel Massih Pio \\ Faculdade de Medicina de Marília, SP, Brasil. \\ Faculdade de Medicina de Marília, SP, Brasil. \\ Thaís Munholi Raccioni \\ Faculdade de Medicina de Marília, SP, Brasil.
}

\begin{abstract}
Resumo: Considerando a escassez de estudos acerca da teoria e prática do Acompanhamento Terapêutico (AT) e um aparente desconhecimento dessa atividade em serviços de saúde mental de um município do interior paulista, este artigo buscou analisar o conhecimento e percepções de equipes de saúde mental acerca dessa temática e discutir a viabilidade local de inclusão deste dispositivo como estratégia de cuidado em saúde mental, dentro da perspectiva da Clínica Ampliada e Reforma Psiquiátrica. $\mathrm{O}$ trabalho foi realizado com profissionais da saúde de diversas áreas, nos seguintes serviços: Centro de Atenção Psicossocial II, Centro de Atenção Psicossocial Álcool e Drogas II, Centro de Atenção Psicossocial Infanto-juvenil II e Enfermaria Psiquiátrica. Para a coleta de dados foi utilizada a abordagem do grupo focal e, para a análise dos dados, $o$ Discurso do Sujeito Coletivo (DSC). A presente pesquisa possibilitou a problematização acerca do tema e a abertura de espaços para viabilizar a construção deste trabalho nos serviços, o qual consiste em uma potente estratégia para a reforma em saúde mental.
\end{abstract}

Palavras-chaves: Reforma Psiquiátrica; Saúde Mental; Clínica Ampliada; Acompanhamento Terapêutico.

\section{Therapeutic Accompaniment: Concepts and Possibilities in Mental Health Services}

\begin{abstract}
Considering thelack of studies on the theoryand practice of Therapeutic Accompaniment (TA) and an apparent unknowledge of this activity in mental health services of a municipality in the interior of São Paulo, this research aimed to analyze the knowledge and perceptions of mental health teams and discuss the local feasibility of including this device as a mental health care strategy, from the perspective of Enlarged Clinic and Psychiatric Reform. The study was accomplished with health professionals from several areas in the following municipal services: Psychosocial Care Center II, Psychosocial Care Center Alcohol and Drugs II, Psychosocial Care Center Children and Youth II and Psychiatric Ward. For data collection was used the approach of the focus group and, for data analysis, the Collective Subject Discourse (CSD). This research enabled the questioning about the subject and the opening of opportunities to enable the construction of this work in the services, which consists in a powerful strategy for mental health reform.
\end{abstract}

Keywords: Psychiatric Reform; Mental health; Enlarged Clinic; Therapeutic Accompaniment. 


\title{
Acompañamiento Terapéutico: Conceptos y Posibilidades en Servicios de Salud Mental
}

\begin{abstract}
Resumén: Considerando la escasez de estudios sobre la teoría y práctica del Acompañamiento Terapéutico (AT) y un aparente desconocimiento de la actividad en servicios mentales de un municipio del interior paulista, ese trabajo ha buscado analizar el conocimiento y percepción de equipes de salud mental acerca del tema y discutir la viabilidad local de inclusión de este dispositivo como estrategia de cuidado en salud mental, dentro de la perspectiva de Clínica Ampliada y Reforma Psiquiátrica. El trabajo fue realizado con profesionales de la salud de diferentes sectores, en los puntos: Centro de Atención Psicosocial II, Centro de Atención Psicosocial Alcohol y Drogas II, Centro de Atención Psicosocial Infante-Juvenil II y Enfermería Psiquiátrica. Para coleta de los datos fue utilizada elabordaje de grupo focal y, para el analicé, el Discurso del Sujeto Colectivo (DSC). La presente pesquisa posibilito la discusión acerca del tema y la abertura de espacios para la posibilidad de construcción de este trabajo en los servicios, el cual consiste en una potente estrategia para la reforma de la salud mental.
\end{abstract}

Palabras clave: Reforma Psiquiátrica; Salud Mental; Clínica Ampliada; Acompañamiento Terapéutico.

\section{Introdução}

A Reforma Psiquiátrica brasileira teve início na década de 1970 - contemporânea ao movimento da Reforma Sanitária - com a emergência das críticas à ineficiência da assistência pública em saúde e ao caráter privatista da política de saúde. A Reforma Psiquiátrica evita a internação como destino, colocando-a como um recurso eventualmente necessário, e agencia o problema social da loucura, permitindo a reinserção social das pessoas com sofrimento mental (Tenório, 2002). Segundo o autor:

As razões pelas quais a psiquiatria não foi capaz de oferecer essa outra resposta começam no mandato social que a legitima (o mandato de reclusão dos loucos), passam pelos valores culturais e sociais mais amplos de segregação da diferença, incluem o caráter refratário ao laço social característico da própria psicose e culminam na dificuldade técnica e gerencial de promover com eficácia e abrangência essa outra resposta (p. 55-56).

$\mathrm{Na}$ atualidade, a Portaria Ministerial no 3.088, de 23 de dezembro de 2011, instituiu a Rede de Atenção Psicossocial (RAPS), destinada a todas as pessoas com sofrimento ou transtorno mental, incluindo aqueles decorrentes do uso prejudicial de substâncias psicoativas, com a finalidade de criação, ampliação e articulação dos pontos de atenção à saúde para essa população (Brasil, 2011). Fazem parte da RAPS os seguintes dispositivos: Estratégia Saúde da Família (ESF), Núcleo de Apoio à Saúde da Família (NASF), Equipe de Consultório de Rua, Centros de Convivência e Cultura, Centros de Atenção Psicossocial (CAPS I, II, III, álcool e drogas e infanto-juvenil), Unidades de Acolhimento Transitório (UAT), Comunidades Terapêuticas, Serviços Residenciais Terapêuticos (SRT), Unidades de Pronto Atendimento (UPA), Serviço de Atendimento Móvel de Urgência (SAMU) e enfermaria especializada em Hospital Geral.

Neste cenário está imerso o cuidado ampliado, que requer mais que a atenção a sintomas e prescrição de tratamentos, no qual os profissionais da atenção psicossocial (psicólogos, terapeutas ocupacionais, enfermeiros, assistentes sociais, médicos) passam a se ocupar dos sujeitos que necessitam desse cuidado, levando em conta aspectos do cotidiano, do tempo, do espaço, do trabalho, do lazer, do ócio, do prazer e da organização de atividades conjuntas, enfatizando as partes mais sadias e as potencialidades do indivíduo (Pitiá,\&Furegato, 2009).

Assim, é dentro do âmbito da Reforma Psiquiátrica que surge o Acompanhamento Terapêutico (AT) ${ }^{1}$, tipo de atendimento clínico caracterizado pela prática

${ }^{1}$ Utiliza-se, como convenção, a denominação AT (Acompanhamento Terapêutico) para a prática clínica, e at (acompanhante terapêutico) quando se tratar do profissional que exerce a prática. 
de saídas pela cidade ou estar ao lado da pessoa com dificuldades psicossociais, buscando auxiliá-la novamente na circulação social, considerando suas limitações e seu contexto histórico (Pitiá,\&Furegato, 2009).

Estellita-Lins, Oliveira e Coutinho (2009) trazem uma síntese acerca da caracterização do trabalho realizado em um AT:

O dia-a-dia, o contexto familiar, social, o lazer vistos de dentro da realidade cotidiana vivenciada nos atendimentos sinalizarão os caminhos a serem percorridos, os objetivos a serem traçados e o término do trabalho, constituindo um impulso para a reconstrução de uma autonomia de base e para a plena utilização dos recursos e potenciais disponíveis no cotidiano do paciente (p. 207).

Nesse sentido, é importante destacar que o profissional não deve tomar decisões pelo sujeito a quem está prestando assistência, mas ser um estímulo norteador nas resoluções de seus conflitos, auxiliando-o em sua independência (Carniel,\& Pedrão, 2010). Desse modo, o AT deve persistir pelo tempo necessário até que o usuário readquira a sua autonomia, se retirando de cena gradualmente nesse processo.

No Brasil, a configuração dessa prática tem início durante a década de 1960, em Porto Alegre, na Clínica Pinel, sob a influência de um trabalho similar que era realizado nas comunidades terapêuticas dos Estados Unidos. Na Clínica Pinel, essa função era exercida principalmente por estudantes de Psicologia e Medicina, como parte de sua formação, assim como por pessoas sem nível superior, recebendo o nome de atendente psiquiátrico, o qual trabalhava em parceria com o psicoterapeuta, buscando colocar em ação os programas de tratamento elaborados pelo mesmo, tanto dentro da instituição, como fora dela (Silva,\& Silva, 2006).

A partir da experiência em Porto Alegre, a prática do AT começa a aparecer em outras regiões do país, como no Rio de Janeiro, ao final de 1969, na Clínica Villa Pinheiros (Silva,\& Silva, 2006), e em São Paulo, no final da década de 1970, no Instituto A Casa (Simões,\&Kirschbaum, 2005). No Rio de Janeiro, a pessoa que exercia a função do AT recebia outro nome, o de auxiliar psiquiátrico, havendo, nesse contexto, uma maior influência da psicanálise do que dos pressupostos da comunidade terapêutica americana (Silva,\& Silva, 2006). Em São Paulo, havia os chamados amigos qualificados, sob influência direta da Argentina (Pitiá,\&Furegato, 2009). Atualmente, os profissio- nais que exercem essa função são denominados de acompanhantes terapêuticos (at).

Dessa forma, a prática e a teorização do AT vêm, desde a década de 1960, crescendo e ocupando espaço nos serviços de saúde mental, sendo que, de início, também acabavam reproduzindo a lógica adaptativa do modelo manicomial, ao manter o controle sobre o paciente para além dos muros da instituição psiquiátrica, tendo uma função de contenção e seguindo um programa claro e predeterminado. Nesse sentido, é fundamental a constante problematização da prática do AT, fazendo com que efetivamente se torne um dispositivo clínico, adquirindo uma função terapêutica (Silva,\& Silva, 2006).

Atualmente, estudos mostram a prática do AT em diversos âmbitos, como na educação inclusiva, em que $o$ at atua como secretário, intérprete e tradutor da ambiência, auxiliando na subjetivação da criança e conexão entre os elementos do território escolar (Sereno, 2006); com pacientes depressivos com risco de suicídio, intervindo na crise, no monitoramento da evolução clínica e contribuindo no processo de reabilitação (Estellita-Lins, Oliveira, Coutinho, 2006) e na alta-assistida após internação hospitalar, visando vincular o usuário em serviço extra-hospitalar, evitar as reinternações hospitalares e inclusão na rede social (Bezerra \& Dimenstein, 2009; Fiorati,\&Saeki, 2008). Na bibliografia estudada, não foram encontrados estudos envolvendo AT e dependência química, consistindo em uma área importante para se pensar e planejar essa prática.

Um último ponto importante a se destacar refere-se à formação dos profissionais que atuam como at, consistindo em uma atividade que não é reconhecida como sendo privativa de alguma área de conhecimento ou profissão (Londero,\& Pacheco, 2006). Assim, diversos profissionais da saúde mental exercem essa prática, trazendo o risco de, muitas vezes, afastarem-se de uma intervenção clínica - que deveria enxergar o usuário como sujeito e buscar a criação de narrativas pessoais e novas formas de estar no mundo - e acabar realizando uma prática baseada em um senso comum, buscando apenas o treinamento e a aquisição de habilidades por parte do paciente (Kirschbaum,\& Rosa, 2003).

A partir da experiência como psicóloga residente em Saúde Mental em um município do interior paulista, foram observados um aparente desconhecimento e uma ausência da prática do AT nos serviços de saúde 
mental. Foi realizada revisão bibliográfica no Portal de Pesquisa da Biblioteca Virtual em Saúde (BVS) utilizando as palavras-chave: acompanhamento terapêutico, psiquiatria, saúde mental e clínica ampliada, e foram encontrados 109 documentos distribuídos nas seguintes bases de dados: Lilacs, BDENF - Enfermagem (Brasil) e Index Psicologia - Periódicos técnico-científicos. A busca abrangeu documentos até o ano de 2013, período em que foi realizada a revisão bibliográfica. Destes, foram escolhidos 33 documentos para aprofundamento, que se relacionavam diretamente à prática do AT dentro do âmbito da saúde mental. Como se pode notar, havia poucos textos diretamente ligados à temática proposta no presente trabalho.

Assim, considerando a realidade local e a aparente escassez de trabalhos específicos relacionados à temática, este trabalho buscou analisar o conhecimento que as equipes de serviços de saúde mental tinham acerca do AT e discutir a viabilidade de inclusão deste dispositivo como estratégia de cuidado em saúde mental, dentro da perspectiva da Clínica Ampliada e Reforma Psiquiátrica.

\section{Metodologia}

\section{Participantes}

A presente pesquisa foi realizada com equipes de serviços de saúde mental de um município de médio porte do interior paulista, o qual conta com os seguintes serviços que oferecem atendimento em saúde mental: 12 Unidades Básicas de Saúde (UBS), 34 Unidades de Saúde da Família (USF), quatro equipes de NASF, duas UPAs, um serviço de psiquiatria no Pronto Socorro, três CAPS (sendo um CAPS II, um CAPS ad II e um CAPS i II), um Ambulatório de Saúde Mental, uma Enfermaria Psiquiátrica em Hospital Geral (a qual possui 18 leitos) e um Hospital Psiquiátrico.

Dentre esses serviços, foram selecionados quatro para a realização da pesquisa, a saber: CAPS II, CAPS ad II, CAPS i II e Enfermaria Psiquiátrica. A amostra intencional envolveu serviços constituintes da RAPS e, consequentemente, que trabalhassem com os pressupostos da Reforma Psiquiátrica, e serviços que trabalhassem especificamente com a saúde mental. Todas as equipes eram multiprofissionais e houve a participação de profissionais de nível superior e nível médio, sendo que, apesar de haver uma predominância de profissionais de nível superior, houve uma equivalência dos dois níveis nos participantes do CAPS i II. Houve um número reduzido de participantes do CAPS ad II, em comparação aos demais serviços, porém trata-se já de uma equipe reduzida, contando com menos profissionais no serviço. Assim, participaram da pesquisa 29 profissionais da saúde, de acordo com a disponibilidade e consentimento de cada participante, sendo que não houve desistências ao longo da pesquisa. O Quadro 1 ilustra o número de participantes, considerando a profissão e o serviço de saúde mental.

\section{Procedimento}

A presente pesquisa é de caráter qualitativo, pois tem como objetivo investigar o conhecimento e as percepções que as equipes possuem acerca AT. A coleta de dados foi realizada no ambiente natural dos participantes, no caso os serviços de saúde acima citados, visando preservar suas características e relações e buscando entender como os fenômenos ocorrem naturalmente e as relações estabelecidas entre eles (Turato, 2013).

Para a coleta de dados, foi realizado um grupo focal com cada equipe dos serviços de saúde mental, totalizando quatro grupos focais durante toda a pesquisa. Segundo Backes, Colomé, Erdmann e Lunardi (2011), é possível pensar o grupo focal como uma técnica de entrevista em grupo, na qual a interação entre os participantes se configura como parte integrante do método, sendo a partir dessa interação que se promove uma ampla problematização acerca de um tema ou foco específico. Nesse sentido, o que se busca é analisar a interação entre os participantes e como os significados são construídos em grupo. (Hernandez Sampieri, Fernandez Collado \& Baptista Lucio, 2013).

Para introduzir o assunto e disparar as discussões, foi elaborado um texto sobre o tema a ser abordado, o AT. Também houve a construção de um roteiro semiestruturado, que guiou o desenvolvimento do grupo. Inicialmente, foram explicados os propósitos da pesquisa e entregue o Termo de Consentimento Livre e Esclarecido ${ }^{2}$. Em seguida, se iniciou o procedimento com a leitura do texto elaborado, seguida da discussão. Através da interação dos participantes, se buscou refletir sobre a temática proposta, contribuindo para o pensar de atitudes, concepções e prá-

${ }^{2} \mathrm{O}$ projeto foi avaliado e aprovado pelo Comitê de Ética em Pesquisa Envolvendo Seres Humanos com Parecer nº 1.012.783. 
Quadro 1

Caracterização dos participantes com relação à profissão.

\begin{tabular}{lccccc}
\hline Profissão & CAPS II & CAPS ad II & CAPS i II & Enfermaria & Total \\
\hline Psicólogo & 1 & 1 & 1 & 1 & 4 \\
Terapeuta Ocupacional & - & - & 1 & 2 & 3 \\
Assistente Social & 1 & 1 & - & 2 & 4 \\
Enfermeiro & 2 & 1 & 2 & 2 & 7 \\
Técnico de Enfermagem & 2 & 1 & 4 & - & 7 \\
Médico & - & - & - & 3 & 3 \\
Fisioterapeuta & 1 & - & - & - & 1 \\
Total & 7 & 4 & 8 & 10 & 29 \\
\hline
\end{tabular}

ticas. Cada grupo focal teve duração de, aproximadamente, uma hora e foram gravados em áudio para posterior transcrição e análise dos dados.

\section{Análise dos dados}

Para a análise dos dados foram utilizados os pressupostos da clínica ampliada e da Reforma Psiquiátrica como referenciais teóricos e, como referencial metodológico, o Discurso do Sujeito Coletivo (DSC). A técnica do DSC consiste em reunir conteúdos de depoimentos individuais com sentidos semelhantes, construindo discursos únicos redigidos na primeira pessoa do singular, de modo a produzir um efeito de "coletividade falando" (Lefévre, Lefévre,\& Marques, 2009). Assim, busca-se reconstituir um sujeito coletivo que, ao mesmo tempo, fala como se fosse indivíduo, mas transmite uma representação com conteúdo ampliado. Desse modo, para a construção do DSC, são utilizados trechos literais dos depoimentos individuais, os quais sinalizam os principais conteúdos das respostas e são denominados de Expressões-chave (ECH) (Lefévre,\&Lefévre, 2006). As ECHs, por sua vez, são representantes das Ideias Centrais (ICs) dos discursos dos participantes, as quais são fórmulas sintéticas que relevam e descrevem os sentidos de cada depoimento (Duarte, Mamede \& Andrade, 2009).

\section{Resultados e Discussão}

O presente estudo buscou identificar as ICs presentes no discurso dos participantes e as ECHs que as representassem, com as quais foram construídos os DSCs. Após a construção dos DSCs, foram identificadas seis temáticas, que foram discutidas de acordo com os temas e discursos de cada serviço de saúde mental. No presente artigo, os discursos não foram apresentados, mas sim as ICs representantes de cada discurso, devido a sua extensão. Assim, os Quadros 2, 3, 4, 5, 6 e 7 trazem as ICs dos discursos, separadas por serviços de saúde, seguidos das respectivas discussões.

Ao iniciar a discussão nos grupos sobre o conhecimento acerca da prática do AT e as experiências relacionadas, foi possível observar um movimento inicial de dúvida, principalmente nos grupos do CAPS i II e da Enfermaria Psiquiátrica (IC4). Nesse primeiro momento, o AT foi relacionado ao acompanhamento do paciente dentro dos serviços de saúde, e não a uma intervenção terapêutica específica (IC 7). Contudo, no decorrer dos grupos, houve a formação de novos conceitos, se aproximando mais de uma prática clínica que realiza intervenções para além do espaço institucional dos serviços de saúde (IC 1, IC 2, IC 3, IC 5, IC 6, IC 8 e IC 9).

Estellita-Lins, Oliveira e Bteshe (2010) situam o desenvolvimento do AT no contexto da Reforma Psiquiátrica, caracterizando-o como uma clínica do espaço domiciliar e do cotidiano, que busca interagir com estressores psicossociais e com os recursos do paciente e de seu entorno.

Aproximando-se dessa clínica do espaço domiciliar e do cotidiano, os discursos do CAPS II e da Enfermaria Psiquiátrica falam sobre uma intervenção mais próxima do paciente, dentro de seu contexto, considerando suas necessidades, subjetividade e grau de autonomia (IC 1 e IC 8). Nesses discursos, o AT aparece como um "acompanhar na vida", como uma intervenção que amplia o trabalho realizado nos serviços, a partir da utilização de outros espaços como a casa e a comunidade.

Em quase todos os discursos apareceram as atividades externas e as visitas domiciliares como apro- 
Quadro 2

Conceitos e experiências associadas ao AT.

\begin{tabular}{|c|c|c|c|}
\hline CAPS II & CAPS ad II & CAPS i II & Enfermaria Psiquiátrica \\
\hline $\begin{array}{l}\text { IC 1: AT como intervenção } \\
\text { no contexto da } \\
\text { pessoa, considerando } \\
\text { suas necessidades e } \\
\text { subjetividade }\end{array}$ & & $\begin{array}{l}\text { IC 4: dúvidas com relação } \\
\text { à teoria e à prática do AT }\end{array}$ & $\begin{array}{c}\text { IC 7: o AT como o } \\
\text { acompanhamento } \\
\text { institucional do paciente, } \\
\text { não para além dos } \\
\text { horizontes do hospital }\end{array}$ \\
\hline $\begin{array}{l}\text { IC 2: experiência em } \\
\text { grupo como possibilidade } \\
\text { de AT }\end{array}$ & $\begin{array}{c}\text { IC 3: experiência de } \\
\text { atividades externas e } \\
\text { visitas domiciliares como } \\
\text { possibilidade de AT }\end{array}$ & $\begin{array}{l}\text { IC 5: experiência de } \\
\text { visitas domiciliares como } \\
\text { possibilidade de AT }\end{array}$ & $\begin{array}{l}\text { IC 8: o AT como } \\
\text { ampliação do trabalho } \\
\text { clínico do hospital, } \\
\text { considerando as } \\
\text { necessidades e o grau de } \\
\text { autonomia do paciente }\end{array}$ \\
\hline & & $\begin{array}{l}\text { IC 6: o AT como uma } \\
\text { intervenção para além do } \\
\text { cenário institucional }\end{array}$ & $\begin{array}{l}\text { IC 9: experiência de } \\
\text { atividades externas como } \\
\text { possibilidade de AT }\end{array}$ \\
\hline
\end{tabular}

ximações de um AT (IC 3, IC 5, IC 6 e IC 9). Com relação às atividades externas, os discursos falam sobre momentos de recreação e de estar em sociedade com o paciente, os quais também consistem em possibilidades de se obter mais informações sobre ele e o seu processo terapêutico. As visitas domiciliares são trazidas como uma forma de se aproximar do paciente e de sua família, sendo também um espaço para colher mais informações sobre sua história e dinâmica familiar. A partir das reflexões realizadas, o CAPS II trouxe a possibilidade da realização do AT em grupo (IC 2), em que, a partir das necessidades identificadas, são propostas atividades em diversos locais na sociedade seguidas de discussões acerca dessas experiências.

É importante destacar que as atividades externas por si só não caracterizam oAT. Énecessário que haja articulação com as demais estratégias de atendimento dos serviços e, assim, que as finalidades do AT tenham como foco as necessidades e subjetividade do paciente. Dessa forma, além da possibilidade de estar com o paciente em outros contextos e colher novas informações, é necessário que essas atividades busquem potencializar a dimensão simbólica do cotidiano do sujeito, possibilitando a ressignificação de suas experiências e novas formas de estar no mundo (Kirschbaum,\& Rosa, 2003).

Ao pensar sobre o profissional que realiza o AT, os grupos não atribuem essa intervenção a uma profissão específica, mas consideram a necessidade de capaci- tação (IC 11 e IC 13), o perfil profissional e também o vínculo e o desejo (IC 10, IC 16 e IC 18), visto que, junto com a capacitação técnica, é necessária uma disponibilidade afetiva para o cuidado em saúde. Londero e Pacheco (2006) falam sobre a inexistência de especialização ou aprimoramento profissional para a prática do AT, trazendo que essa atividade ainda não é reconhecida como privativa de alguma área de conhecimento ou profissão. Nesse sentido, apontam para a urgência da construção de cursos de capacitação para profissionais da área da saúde, visando uma melhor qualificação do cuidado oferecido aos pacientes, além de contribuir para uma futura regulamentação profissional do at.

Outro conceito que apareceu em todos os grupos - com exceção do CAPS i II - foi a possibilidade de familiares assumirem o papel de at (IC 12, IC 15 e IC 17). As equipes pensaram na atuação do familiar como uma forma de dar continuidade ao cuidado oferecido na instituição, também colocando a necessidade de capacitação. Porém, refletem sobre a viabilidade dessa proposta, ao considerar a questão do sofrimento familiar na convivência com o transtorno mental.

Dentro dessa reflexão, é importante retomar a questão da neutralidade terapêutica, em que, de acordo com Sampaio (2004), "Para experienciar e descrever o que acontece no espaço terapêutico, o terapeuta precisa utilizar suas próprias funções de contato. Para tanto, é preciso que tenha suas funções de 
Quadro 3

Quem é o at?

\begin{tabular}{llll}
\hline CAPS II & CAPS ad II & CAPS i II & Enfermaria Psiquiátrica
\end{tabular}

IC 10: $\mathrm{o}$ at pode ser qualquer profissional, considerando o vínculo, o desejo e o compromisso

IC 11: o at deve ter capacitação em saúde mental sensibilizado para desenvolver o AT
IC 13: o at é um profissional de saúde ou alguém com formação para realizar atendimentos terapêuticos

IC 14: o at como um cuidador fora da internação
IC 16: 0 at pode ser qualquer profissional, considerando o vínculo e a necessidade do paciente
IC 17: $\mathrm{o}$ at pode ser qualquer profissional e até um familiar

IC 18: o at é o profissional mais próximo do paciente, considerando o vínculo, o perfil e a necessidade de capacitação
IC 15: o familiar com perfil para ser at, considerandose especificidades da dependência química contato desobstruídas. Assim, o terapeuta pode manter seu foco no cliente" (p.54).

Nas intervenções feitas pelo at, assim como em qualquer intervenção terapêutica, o foco está no paciente e é fundamental que o profissional de saúde mantenha certa neutralidade. A partir disso, é possível pensar sobre a dificuldade de haver essa neutralidade na relação familiar-paciente. O familiar traz vivências e sentimentos com relação ao paciente e seu adoecimento, geralmente presta cuidados ao mesmo e apresenta certa sobrecarga. Desse modo, não há um contato desobstruído, impossibilitando a atuação do familiar como at.

Contudo, o trabalho do at pode contribuir com as questões familiares de outras formas. Nesse sentido, Londero e Pachero (2006) falam sobre a facilitação do convívio entre os membros, buscando a reorganização de papéis e a aquisição de novos repertórios, em que o at serviria como modelo de identificação, auxiliando no manejo do paciente. Azevedo e Dimenstein (2008) ressaltam ainda a importância das estratégias da Reforma Psiquiátrica contemplarem as demandas dos familiares para além do cuidar dos pacientes, com um olhar sobre suas necessidades.

Um último conceito que surgiu, apenas no CAPS ad II, refere-se ao at como alguém que acompanha o paciente 24 horas por dia, auxiliando-o em cuidados mais básicos, como higiene, alimentação e medicação (IC 14). Reis Neto, Pinto e Oliveira (2011) retomam a história do AT, no início como uma forma de substituição da internação, caracterizado por um cuidado mais intensivo até a remissão da crise, mas que, no decorrer do tempo, foi se configurando de outras formas. Desse modo, esse conceito trazido pelo CAPS ad II aproximou o at tanto dessas funções iniciais, do cuidado substitutivo da internação, quanto da figura do cuidador de doenças crônicas, pensando nesse cuidado intensivo e mais voltado para questões práticas do cotidiano. Vale ressaltar que, atualmente, o AT consiste em um trabalho mais amplo, que busca a elaboração e ressignificação de questões internas, a partir da exploração dos espaços sociais onde se intervém.

Com relação às indicações para realização do AT, todos os serviços falam sobre pacientes com maiores prejuízos, pensando em limitações sociais, psíquicas, cognitivas, neurológicas e comportamentais. Contudo, além da gravidade do transtorno, a Enfermaria Psiquiátrica fala sobre o desejo do paciente de ser acompanhado pelo at, colocando-o como participante ativo de seu tratamento (IC 22).

Pitiá e Santos (2006) fizeram uma pesquisa bibliográfica acerca da produção científica na área de AT e o trazem como uma prática clínica voltada para pacientes psicóticos, portadores de deficiência, drogadição e para depressão e risco suicida. Dessa forma, a gravidade do transtorno e as limitações decorrentes são critérios que aparecem na literatura. 
Palombini (2009) discute um aspecto mais específico, em que o AT é solicitado nos casos em que há maiores dificuldades com relação à circulação pelas vias públicas, nos quais o sujeito se mantém em ambientes mais restritos, como o quarto. Cita seu artigo "Passagens obstruídas: quartos privativos, mínimas janelas" (Palombini, 2002), no qual fala que, nesses casos, é preciso primeiro ocupar o espaço do quarto, explorando possibilidades e lugares psíquicos, para depois se adentrar nos espaços da cidade.

Reis Neto, Pinto e Oliveira (2011) colocam mais um aspecto a se refletir:

A prática do acompanhamento terapêutico (AT) em foco neste trabalho surge como prática destinada àqueles pacientes acometidos por transtornos mentais ou por sofrimento psíquico que, por razões variadas, requerem mais (ou menos) do que oferecem os espaços tradicionalmente destinados ao seu tratamento, ou seja: clínicas, hospitais psiquiátricos e consultórios (p. 31).

Nesse trecho, os autores trazem duas indicações para o AT, que podem ser pensadas da seguinte forma: (1) quando, devido à gravidade do transtorno, o atendi- mento oferecido dentro do espaço institucional se torna insuficiente e há a necessidade de buscar outras formas de intervenção para complementar o tratamento; e (2) quando o paciente apresenta maiores recursos internos, necessitando menos do espaço institucional e podendo realizar seu tratamento mais próximo da comunidade e de espaços sociais. Com relação a esta última indicação, Wachs, Jardim, Paulon e Resende (2010) relatam uma experiência de desinstitucionalização de moradores de um Hospital Psiquiátrico a partir da transição para os serviços de residências terapêuticas, utilizando o AT. Os autores refletem sobre a dificuldade em optar entre $o$ olhar vigilante do cuidado tutelar do hospital e a autonomia promovida em um serviço residencial terapêutico, mas que, através do AT, foi possível sustentar essa transição para aqueles que já não precisavam viver dentro da instituição e apresentavam condições e desejo de buscar essa mudança.

Assim, os critérios e o planejamento do AT (número de encontros e duração dos mesmos) deverão considerar tanto as limitações quanto os recursos do paciente, assim como o objetivo terapêutico, o qual é planejado de forma singular para cada caso e norteará as ações tanto dentro do espaço institucional como nos espaços sociais onde o at circulará com o paciente.

\section{Quadro 4}

Indicações para realização do AT.

\begin{tabular}{llll}
\hline CAPS II & CAPS ad II & CAPS i II & Enfermaria Psiquiátrica \\
\hline
\end{tabular}

IC 19: o AT é indicado para pacientes com limitações psíquicas e sociais
IC 20: o AT é indicado para pacientes com limitações neurológicas, cognitivocomportamentais e sociais, com um longo histórico de recaídas e internações
IC 21: o AT é indicado para crianças avaliadas com transtorno mais grave
IC 22: o AT é indicado para pacientes com limitações psíquicas e sociais, considerando seu desejo

Quadro 5

Objetivos do AT.

\begin{tabular}{|c|c|c|c|}
\hline CAPS II & CAPS ad II & CAPS i II & Enfermaria Psiquiátrica \\
\hline $\begin{array}{l}\text { IC 23: o objetivo do AT é } \\
\text { despertar habilidades e } \\
\text { promover autonomia }\end{array}$ & $\begin{array}{l}\text { IC 24: o objetivo do } \\
\text { AT é criar habilidades, } \\
\text { considerando a } \\
\text { autonomia do sujeito }\end{array}$ & $\begin{array}{c}\text { IC 25: o objetivo do AT } \\
\text { é favorecer o vínculo do } \\
\text { paciente com o serviço e } \\
\text { avaliar a dinâmica familiar } \\
\text { IC 26: o objetivo do AT } \\
\text { é reinserir o paciente } \\
\text { na sociedade }\end{array}$ & $\begin{array}{l}\text { IC 27: o objetivo do AT é } \\
\text { reinserir o paciente na } \\
\text { sociedade, desenvolvendo } \\
\text { gradativamente sua } \\
\text { autonomia }\end{array}$ \\
\hline
\end{tabular}


Os discursos sobre os objetivos do AT apresentam o enfoque sobre o desenvolvimento de habilidades, promoção de autonomia e reinserção social (IC 23, IC 24, IC 26 e IC 27). O CAPS II e o CAPS i II falam também sobre o fortalecimento do vínculo com a família e sobre a avaliação da dinâmica familiar (IC 25).

Segundo Pitiá e Santos (2006), "O objetivo terapêutico no AT é descrito como significar aquilo que o sujeito expressa com seu adoecer, para que interaja de forma mais saudável em termos biopsicossociais" (p.2). Assim, ao acompanhar o paciente em seus espaços sociais, $o$ at $o$ auxilia a entrar em contato com suas questões internas e a elaborar novos projetos de vida, a partir do reconhecimento de suas dificuldades e recursos, de intervenções in loco e da sustentação desse processo com a sua presença, enquanto ela for necessária.

Especificando mais os objetivos, Reis Neto, Pinto e Oliveira (2011) falam sobre o AT com pacientes psicóticos, deprimidos e dependentes químicos. Com pacientes psicóticos, citam que o AT pode facilitar a criação de novos laços sociais e afetivos, a partir da escuta à singularidade do delírio e do deslocamento compartilhado pelo espaço público. Com pacientes deprimidos, colocam a presença do at como fator de proteção, no caso de risco de suicídio, e importante para servir às necessidades de atenção do paciente e oferecer possibilidades de troca, considerando que o isolamento social é um sintoma característico da depressão. Na dependência química, o at pode auxiliar o paciente a resistir à vontade de usar a droga e, junto com essa função, há uma série de trocas significativas que se estabelecem entre at e paciente, com a possibilidade da ressignificação da droga na vida do sujeito, assim como da exploração de novas possibilidades no meio social e de vínculos.

Por fim, os autores falam sobre um objetivo comum do AT nos três casos, que seria o de evitar a internação, considerando que é um dispositivo extra-hospitalar "capaz de oferecer uma contenção simbólica àquilo de excessivo que poderia levar à internação" (Reis Neto, Pinto \& Oliveira, 2011, p. 33). Porém, ressaltam que essa não é a sua única finalidade, considerando que o AT não trabalha apenas com a evitação de algo, como a internação ou agravo dos sintomas, mas também com a construção e exploração de novas possibilidades e lugares psíquicos, como já comentado anteriormente.

Após a reflexão e construção de conceitos referentes ao AT, buscou-se aproximar essa prática da realidade dos serviços, pensando se seria uma intervenção viável ou não. O CAPS II e o CAPS i II trazem como algo possível de ser implementado (IC 28 e IC 30), em que o CAPS II relata algumas experiências com AT e ambos os serviços falam sobre o atendimento integral ao paciente. O CAPS ad II e a Enfermaria Psiquiátrica trazem mais dificuldades, as quais envolvem questões de recursos humanos e financeiros, políticas de saúde e desconhecimento dessa atividade, acreditando não ser uma intervenção viável no momento (IC 29 e IC 31).

Na saúde pública brasileira, muitos serviços ainda contam com equipes reduzidas e falta de recursos financeiros, o que realmente dificulta o atendimento à saúde. A ideia que a Enfermaria Psiquiátrica traz com relação ao AT é de uma intervenção cara, o que enfrentaria maiores dificuldades para implementação, pensando na realidade da saúde do país. Contudo, talvez o AT não precise ser visto como uma intervenção tão diferente dos demais atendimentos em saúde, em que também há uma necessidade de capacitação e dedicação de tempo da equipe. O tempo que o profissional de saúde despenderá com o AT não precisa se exceder ao das demais intervenções, sendo também importante colocar que os critérios para realização do AT serão avaliados pela equipe, não sendo, assim, uma intervenção a ser feita com todos os pacientes.

Além dessas dificuldades, o CAPS ad II traz a necessidade de uma intervenção a nível macro, da criação

Quadro 6

AT: uma intervenção possível?

\begin{tabular}{lccc}
\hline \multicolumn{1}{c}{ CAPS II } & CAPS ad II & CAPS i II & Enfermaria Psiquiátrica \\
\hline $\begin{array}{l}\text { IC 28: o AT não é uma } \\
\text { prioridade, mas pode ser } \\
\text { implementado e atender } \\
\begin{array}{l}\text { o indivíduo em sua } \\
\text { integralidade }\end{array}\end{array}$ & $\begin{array}{c}\text { IC 29: necessidade de uma } \\
\text { política de saúde para } \\
\text { implementação do AT, } \\
\text { considerando a falta de } \\
\text { recursos humanos local }\end{array}$ & $\begin{array}{c}\text { IC 30: o AT como uma } \\
\text { intervenção possível e } \\
\text { necessária, pensando na } \\
\text { integralidade do paciente }\end{array}$ & $\begin{array}{c}\text { IC 31: o AT como } \\
\text { untervenção de } \\
\text { difícil acesso }\end{array}$ \\
\hline
\end{tabular}


de uma política de saúde que consolide essa prática e o profissional at (IC 29). Realmente não há uma política de saúde nesse sentido, mas há os pressupostos da Reforma Psiquiátrica e Clínica Ampliada como pano de fundo dos atendimentos em saúde mental, em que o AT se insere, pensando em atendimento extra-hospitalar e em novas formas de intervir em saúde.

Segundo Merhy (2002), "todos os atores sociais que estão no cenário, compondo a situação, também sabem governar, isto é, também têm saberes que lhes permitem um agir governamental" (p.50). Em seu texto, o autor cita Carlos Matus (1987) e coloca que, em uma organização, todos governam - ainda que em diferentes posições e espaços - e atuam em mão dupla, em que a situação institucional define os atores, mas suas ações também definem a situação. Mesmo sendo importante a criação e implementação de políticas públicas de saúde que regulamentem práticas e melhorem a assistência, também são possíveis ações a nível local, com os recursos disponíveis e de acordo com as propostas dos serviços, considerando que os profissionais de saúde também apresentam governabilidade para propor e realizar mudanças, ainda que não em uma posição de alta direção.

Desse modo, se a proposta do serviço visar a implementação do AT, ações a nível macro somariam recursos, mas a ausência das mesmas não impede que o serviço se organize e busque outros recursos (como conhecimento, trabalhadores e comunicação) para concretização da proposta, o que, por sua vez, poderia contribuir para o desenvolvimento das ações a nível macro, a partir da avaliação de seus resultados. A Enfermaria Psiquiátrica fala sobre o AT como uma intervenção de difícil acesso, trazendo a necessidade de articulação com outros setores e, nesse sentido, se coloca como ator na proposta de fazer essa articulação com a rede, buscando refletir sobre essa prática, promover um maior conhecimento e aproximá-la do cotidiano dos serviços.

Por outro lado, ao pensar o AT como uma prática viável - e até necessária - de ser implementada nos serviços, tanto o CAPS II como o CAPS i II falam sobre a não fragmentação do paciente e o acompanhamento em sua integralidade (IC 28 e IC 30). Mattos (2001) traz que a integralidade implica em uma recusa ao reducionismo e à objetivação dos sujeitos, ou seja, olhar para o paciente para além do sintoma, se atentando também para sua subjetividade e demais necessidades de saúde. Além disso, o autor coloca que a integralidade também envolve uma organização contínua do processo de trabalho nos serviços de saúde, a qual busca ampliar as possibilidades de compreensão das necessidades de saúde de uma determinada população e que, para tanto, há a necessidade de diálogo entre os diferentes sujeitos e suas percepções acerca das necessidades dos serviços de saúde.

Assim, os serviços falam sobre o AT como uma possibilidade de acompanhar o paciente em todo o seu espaço transitado e trazem também o envolvimento de toda a rede de suporte disponível. O estar com o paciente em diferentes espaços possibilita ao profissional uma ampliação do olhar e a observação de outras necessidades de saúde, visto que os atendimentos na rua implicam em uma diversidade e proximidade maior de relações e intercorrências. Dessa forma, veem no AT uma ampliação de uma avaliação e acompanhamento, mas também colocam a necessidade do envolvimento de outros dispositivos de cuidado, visto que, ao considerar as diferentes necessidades de saúde, são necessários também o diálogo e a articulação com outros setores e serviços.

Durante o desenvolvimento dos grupos, também foi discutido aspectos do trabalho em rede, o que possibilitou ampliar as condições de realização do AT. Todos os serviços trazem conceitos semelhantes, enfatizando a necessidade do trabalho em rede e intersetorial (IC 33 e IC 35), e apontam algumas dificuldades nesse sentido, como a falta de articulação e comunicação entre os serviços (IC 32 e IC 34). Também trazem novamente a questão da integralidade, colocando a ideia de complementaridade de um ser-

Quadro 7

Desafios do trabalho em rede.

\begin{tabular}{lccc}
\hline CAPS II & CAPS ad II & CAPS i II & Enfermaria Psiquiátrica \\
\hline IC 32: necessidade de & IC 33: necessidade do & IC 34: necessidade de \\
implicação, integração & maior comunicação \\
e comunicação entre os & entre os serviços & IC 35: importância da \\
serviços da rede & intersetorial & $\begin{array}{c}\text { articulação da rede } \\
\text { de cuidados para a } \\
\text { saúde mental }\end{array}$ \\
\hline
\end{tabular}


viço para o outro e, assim como a Enfermaria Psiquiátrica diz que o paciente na verdade circulará fora do hospital, os CAPS também falam sobre essa transitoriedade do paciente junto aos serviços, em que o objetivo final é a reintegração à sociedade.

Como já mencionado, a Portaria Ministerial $n^{\circ}$ 3.088, de 23 de dezembro de 2011 (Brasil, 2011), instituiu a RAPS, onde são descritos os principais serviços e ações em atenção psicossocial no país para pessoas portadoras de transtornos mentais e usuárias de substâncias psicoativas. Apesar de não contar com todos os dispositivos constituintes da RAPS, o município pesquisado possui uma rede de saúde mental, contemplando serviços e ações preconizados pela regulamentação. Talvez uma melhor estruturação desses serviços auxiliaria no trabalho em rede, mas os grupos falam principalmente sobre dificuldades referentes a recursos humanos.

Bedin (2010) cita a carência e a fragmentação dos serviços de rede substitutivos aos hospitais psiquiátricos, trazendo a dificuldade da falta de articulação entre os serviços, os quais nem sempre trabalham na lógica da continuidade do cuidado, mas na lógica do encaminhamento e da falta de corresponsabilização. Desse modo, há a necessidade do envolvimento de todos na garantia do cuidado em saúde. Como já mencionado anteriormente, o AT se insere nessas novas práticas que a clínica ampliada possibilita aos profissionais de saúde e, apesar do foco da presente pesquisa ter sido o AT, a ideia também era de que, a partir da reflexão proposta, os serviços pudessem inventar novas práticas, de acordo com suas próprias necessidades.

Nesse sentido, é possível pensar que as dificuldades do trabalho em rede - seja por recursos humanos ou estruturais - refletem também uma dificuldade para a implementação efetiva do cuidado extra-hospitalar, em que temos o AT como uma das estratégias. Assim, é a partir do fortalecimento dos dispositivos extra-hospitalares e de um trabalho em rede efetivo que será possível a diminuição das intervenções hospitalares e a promoção do cuidado em espaços de liberdade.

\section{Considerações finais}

Apesar do AT ainda não ser uma prática muito utilizada nos serviços e, em alguns, até ser algo desconhecido, todos os grupos conseguiram refletir sobre o tema, construir conceitos e se aproximar desse tipo de intervenção.

Algo importante a ser colocado refere-se ao potencial de ação que os serviços apresentam, mas que, muitas vezes, não conseguem viabilizar. Os serviços parti- cipantes da pesquisa trazem empecilhos para cumprir o proposto pela reforma em rede, especialmente no que se refere à exploração de seus potenciais extra-hospitalares e das possibilidades existentes no território.

Com relação ao AT, as maiores dificuldades atrelam-se à capacitação profissional (a qual envolve não apenas conhecimento, mas também habilidades e atitudes relacionadas à formação), a dificuldades internas, em que falam principalmente sobre a falta de recursos humanos, e a dificuldades externas, que se referem à necessidade de comunicação entre os serviços da rede e de novas políticas públicas de saúde.

Embora muito tenha se conseguido com relação à Reforma Psiquiátrica brasileira, seu movimento ainda é recente e a luta por um cuidado mais humanizado, significativo e efetivo em saúde mental continua. Assim, apesar de atualmente se poder contar com uma rede de serviços substitutivos aos hospitais psiquiátricos, essa rede e muitos desses serviços ainda estão em construção. Desse modo, a forma de cuidar nesses serviços também está em desenvolvimento, o que reflete os cuidados muitas vezes mais ambulatoriais, dentro da instituição, e não explorando outros espaços na comunidade.

Durante os grupos, não foi possível entrar diretamente nessa discussão e se trabalhou mais com o que os serviços conheciam e pensavam sobre o AT. Contudo, considerando que o AT carrega consigo os pressupostos da Reforma Psiquiátrica e o trabalho no território é o que o caracteriza, é possível pensar que alguma reflexão nesse sentido possa ter se iniciado.

Assim, apesar de o trabalho ter se direcionado a uma intervenção extra-hospitalar específica, é importante não perder de vista essa discussão mais ampla, que se refere à transição da lógica hospitalocêntrica para uma estrutura de serviços de base territorial, em que é preciso se atentar para as dificuldades ainda presentes nesse processo, as quais interferem no funcionamento da rede de saúde mental e, consequentemente, na implementação do cuidado extra-hospitalar.

Por fim, a presente pesquisa também buscou contribuir com a literatura e conhecimento referente ao AT, considerando a escassez de trabalhos encontrados e o desconhecimento dessa prática entre os profissionais de saúde. Apesar das dificuldades apontadas, um espaço foi criado para viabilizar a construção deste trabalho nos serviços, o qual se apresenta como uma potente estratégia para a reforma em saúde mental. Ao final, foi apresentado aos serviços os resultados 
deste estudo, sendo entregue uma cópia do trabalho para cada equipe e para a Secretaria Municipal de Saúde do município, buscando compartilhar a experiência e conhecimento desenvolvidos com a pesquisa e favorecendo novas possibilidades nos serviços.
Ainda se fazem necessários novos estudos envolvendo o tema e a prática do AT, buscando enriquecer a literatura na área, assim como fortalecer a efetivação dessa estratégia em serviços de saúde mental, dentro do âmbito da Reforma Psiquiátrica.

\section{Referências}

Azevedo, T., \& Dimenstein, M. (2008). O acompanhamento terapêutico no cuidado em saúde mental. Estudos e Pesquisas em Psicologia, 33, 658-671. Recuperado de http://www.e-publicacoes.uerj.br/index.php/ revispsi/article/view/10553

Backes, D. S., Colomé, J. S., Erdmann, R. H., \&Lunardi, V. L. (2011).Grupo focal como técnica de coleta e análise de dados em pesquisas qualitativas. O Mundo da Saúde, 35, 438-442. Recuperado de http://bvsms.saude.gov.br/ bvs/artigos/grupo_focal_como_tecnica_coleta_analise_dados_pesquisa_qualitativa.pdf

Bedin, D. M. (2010). Passos e descompassos: práticas em saúde mental na perspectiva da integralidade (Dissertação de mestrado). Pontifícia Universidade Católica do Rio Grande do Sul, Porto Alegre, RS.

Bezerra, C. G., \& Dimenstein, M. (2009). Acompanhamento terapêutico na proposta de alta-assistida implementada em hospital psiquiátrico: relato de uma experiência. Psicologia Clínica, 21(1), 15-32. https:// doi.org/10.1590/S0103-56652009000100002

Brasil. Ministério da Saúde (2011). Portaria No 3.088, de 23 de dezembro de 2011. Institui a Rede de Atenção Psicossocial para pessoas com sofrimento ou transtorno mental e com necessidades decorrentes do uso de crack, álcool e outras drogas, no âmbito do Sistema Único de Saúde (SUS). Brasília, DF: o autor.

Carniel, A. C. D., \& Pedrão, L. J. (2010). Contribuições do acompanhamento terapêutico na assistência ao portador de transtorno mental. Revista Eletrônica de Enfermagem, 12(1), 63-72. Recuperado de http://www.fen.ufg.br/ revista/v12/n1/v12nla08.htm

Duarte, S. J. H., Mamede, M. V., \& Andrade, S. M. O. (2009). Opções teórico-metodológicas em pesquisas qualitativas: representações sociais e discurso do sujeito coletivo. Saúde e Sociedade, 18(4), 620-626. https://doi.org/10.1590/S0104-12902009000400006

Estellita-Lins, C., Oliveira, V. M., \&Bteshe, M. (2009). Por uma tentativa de situar o Acompanhamento Terapêutico entre a psicanálise e a psiquiatria comunitária. Adverbum, 4(2), 59-63. Recuperado de https://www.arca.fiocruz. $\mathrm{br} /$ handle/icict/859

Estellita-Lins, C., Oliveira, V. M. \& Coutinho, M. F. (2006). Acompanhamento terapêutico: intervenção sobre a depressão e o suicídio. Psyche (São Paulo), 10(18), 151-166. Recuperado de http://pepsic.bvsalud.org/scielo. php?script=sci_arttext\&pid=S1415-11382006000200015\#1

Estellita-Lins, C., Oliveira, V. M., \& Coutinho, M. F. (2009). Clínica ampliada em saúde mental: cuidar e suposição de saber no acompanhamento terapêutico. Ciência \& Saúde Coletiva, 14(1), 205-215. https://doi.org/10.1590/S1413-81232009000100026

Fiorati, R. C., \&Saeki, T. (2008). O acompanhamento terapêutico na internação hospitalar: inclusão social, resgate de cidadania e respeito à singularidade. Interface (Botucatu), 12(27), 763-772. https:// doi.org/10.1590/S1414-32832008000400007

Hernandez Sampieri, R., Fernandez Collado, C., \& Baptista Lucio, P. (2013) Metodologia de pesquisa. Porto Alegre, RS: Penso.

Kirschbaum, D. I. R., \& Rosa, T. M. (2003). Os trabalhadores de enfermagem como acompanhantes terapêuticos de um centro de atenção psicossocial. Revista da Escola de Enfermagem da USP, 37(1), 97-106. https:// doi.org/10.1590/S0080-62342003000100012

Lefévre, F., \&Lefévre, A. M. C. (2006). O sujeito coletivo que fala. Interface (Botucatu), 10(20), 517-524. https://doi. org/10.1590/S1414-32832006000200017.

Lefévre, F., Lefévre, A. M. C., \& Marques, M. C. C. (2009). Discurso do sujeito coletivo, complexidade e organização. Ciência \& Saúde Coletiva, 14(4), 1193-1204. https://doi.org/10.1590/S1413-81232009000400025 
Londero, I.,\& Pacheco, J. T. B. (2006). Por que encaminhar ao acompanhante terapêutico? Uma discussão considerando a perspectiva de psicólogos e psiquiatras. Psicologia em Estudo, 11(2), 259-267. https://doi.org/10.1590/S1413-73722006000200004

Mattos, R. A. (2001). Os sentidos da integralidade: algumas reflexões acerca dos valores que merecem ser defendidos. In R.Pinheiro, \& R. A. Mattos (Orgs.), Os sentidos da integralidade na atenção e no cuidado à saúde (pp. 39-64). Rio de Janeiro: Abrasco.

Merhy, E. E. (2002). Introdução ao debate sobre os componentes da caixa de ferramentas dos gestores em saúde. In Brasil. Ministério da Saúde. Secretaria de Gestão do Trabalho e Educação na Saúde. Ver-SUS Brasil: Vivências e estágios na realidade do SUS do Brasil (pp. 48-62). Brasília, DF: o autor.

Palombini, A. L. (2002). Passagens obstruídas: quartos privativos, mínimas janelas. Revista da Associação Psicanalítica de Porto Alegre. Clínica da Adolescência 23, 63-68. Recuperado de http://www.appoa.com.br/uploads/ arquivos/revistas/revista23.pdf

Palombini, A. L. (2009). Utópicas cidades de nossas andanças: flânerie e amizade no acompanhamento terapêutico. Fractal: Revista de Psicologia, 21(2), 295-318. https://doi.org/10.1590/S1984-02922009000200008

Pitiá, A. C. A., \&Furegato, A. R. F. (2009). O Acompanhamento Terapêutico (AT): dispositivo de atenção psicossocial em saúde mental. Interface (Botucatu), 13(30), 67-77. https://doi.org/10.1590/S1414-32832009000300007

Pitiá, A. C. A., \& Santos, M. A. (2006). O acompanhamento terapêutico como estratégia de continência do sofrimento psíquico. SMAD, Revista Eletrônica Saúde Mental Álcool e Drogas, 2(2), 1-14. Recuperado de http://pepsic.bvsalud.org/scielo.php?pid=S1806-69762006000200008\&script=sci_arttext

Reis Neto, R. O., Pinto, A. C. T., \& Oliveira, L. G. A. (2011). Acompanhamento terapêutico: história, clínica e saber. Psicologia: Ciência e Profissão, 31(1), 30-39. https:// doi.org/10.1590/S1414-98932011000100004

Sampaio, M. M. A. (2004). Neutralidade na relação terapêutica: reflexões a partir da abordagem gestáltica. Arquivos Brasileiros de Psicologia, 56(1), 49-56. Recuperado de http://pepsic.bvsalud.org/scielo.php?script=sci_arttext\&pid=S1809-52672004000100005

Sereno, D. (2006). Acompanhamento terapêutico e educação inclusiva. Psyche (São Paulo), 10(1), 167-179. Recuperado de http://pepsic.bvsalud.org/scielo.php?script=sci_arttext\&pid=S1415-11382006000200016

Silva, A. S. T., \& Silva, R. N. (2006). A emergência do acompanhamento terapêutico e as políticas de saúde mental. Psicologia: Ciência e Profissão, 26(2), 210-221. httpd://doi.org/10.1590/S1414-98932006000200005

Simões, C. H. D., \&Kirschbaum D. I. R. (2005). Produção científica sobre o acompanhamento terapêutico no Brasil de 1960 a 2003: uma análise crítica. Revista Gaúcha de Enfermagem, 26(3), 392-402. Recuperado de http://www. seer.ufrgs.br/RevistaGauchadeEnfermagem/article/viewArticle/4569

Tenório, F. (2002). A reforma psiquiátrica brasileira, da década de 1980 aos dias atuais: história e conceito. História, Ciências, Saúde-Manguinhos,9(1), 25-59. https://doi.org/10.1590/S0104-59702002000100003

Turato, E. R. (2013). Clarificando para o empreendimento da pesquisa clínico-qualitativa. In E. R. Turato, Tratado da metodologia da pesquisa clínico-qualitativa: construção teórico-epistemológica, discussão comparada e aplicação nas áreas da saúde e humanas (6a ed., pp. 245-303). Petrópolis, RJ: Vozes.

Wachs, F., Jardim, C., Paulon, S. M., \& Resende, V. (2010). Processos de subjetivação e territórios de vida: o trabalho de transição do hospital psiquiátrico para serviços residenciais terapêuticos. Physis, 20(3), 895-912. https://doi.org/10.1590/S0103-73312010000300011

\section{Amanda Candeloro Cunha}

Graduação em Psicologia pela Universidade Federal de São Carlos (UFSCar) e pós-graduação em Saúde Mental pela Faculdade de Medicina de Marília (Famema), na modalidade de Residência Integrada Multiprofissional em Saúde.E-mail: amanda.candeloro@gmail.com

\section{Danielle Abdel Massih Pio}

Psicóloga. Assistente de Ensino (Famema); Coordenadora do Programa de Residência Integrada Multiprofissional em Saúde Mental (Famema); Doutora em Saúde Coletiva na Unesp/Botucatu. E-mail: danimassihpio@hotmail.com 
Thaís Munholi Raccioni

Graduação em Terapia Ocupacional pela Universidade Estadual Paulista "Júlio de Mesquita Filho" (Unesp) e pós-graduação em Saúde Mental pela Faculdade de Medicina de Marília (Famema), na modalidade de Residência Integrada Multiprofissional em Saúde. E-mail: thaisinha210@hotmail.com

Endereço para envio de correspondência:

Rua Américo Vespúcio, 23 - Jardim Alvorada. CEP: 17513-340 Marília, SP, Brasil

Recebido: 10/01/2016

Reformulado: $10 / 05 / 2017$

Aprovado: 02/08/2017

Received:01/10/2016

Reformulated: $05 / 10 / 2017$

Approved: 08/02/2017

Recebido: 10/01/2016

Reformulado: $10 / 05 / 2017$

Aceptado: 02/08/2017

Como citar: Cunha, A. C., Pio, D. A. M., \& Raccioni, T. M. (2017). Acompanhamento terapêutico: concepções e possibilidades em serviços de saúde mental. Psicologia: Ciência e Profissão, 37(3), 638-651. https://doi.org/10.1590/1982-3703000092016

How to cite: Cunha, A. C., Pio, D. A. M., \& Raccioni, T. M. (2017). Therapeutic accompaniment: concepts and possibilities in mental health services. Psicologia: Ciência e Profissão, 37(3), 638-651. https://doi.org/10.1590/1982-3703000092016

Cómo citar: Cunha, A. C., Pio, D. A. M., \& Raccioni, T. M. (2017). Acompañamiento terapéutico: conceptos y posibilidades en servicios de salud mental. Psicologia: Ciência e Profissão, 37(3), 638-651. https://doi.org/10.1590/1982-3703000092016 